\title{
Crossover to surface flow in supercooled unentangled polymer films
}

\author{
Chi-Hang Lam* \\ Department of Applied Physics, Hong Kong Polytechnic University, Hung Hom, Hong Kong, China \\ Ophelia K. C. Tsui ${ }^{\dagger}$ \\ Department of Physics, Boston University, Boston, Massachusetts 02215, USA \\ (Received 1 August 2013; published 16 October 2013)
}

\begin{abstract}
We study the driven flow of an unentangled glassy polymer film with a free upper surface and supported below by a substrate using nonequilibrium molecular dynamics simulations based on a bead-spring model. Above the glass transition temperature $T_{g}$, simple Poiseuille laminar flow is observed with the film mobility defined as the flow current density per unit pressure gradient scaling as $h^{3}$ with the film thickness $h$. Below $T_{g}$, the film mobility becomes independent of $h$, signifying surface transport. This is in full agreement with recent experiments on the time evolution of capillary waves in polystyrene films supported by silica. A mobile layer is found responsible for the surface transport, as previously conjectured. Our result also shows that it has a velocity profile decaying exponentially into the bulk.
\end{abstract}

DOI: 10.1103/PhysRevE.88.042604

PACS number(s): 83.80.Sg, 64.70.pj, 61.20.Ja, 47.15.gm

\section{INTRODUCTION}

Glassy polymer films are of great technological importance. They also play an important role in studying the general dynamics of glass under confined geometries [1]. For a long time, the glass transition temperature $T_{g}$ of thin polymer films supported by a substrate or free standing has been observed to depend nontrivially on the film thickness [2,3]. One of the major reasons is believed to be the existence of a thin layer at the free surface with enhanced mobility $[2,4]$. This notion is supported by the strong heterogeneity in the local dynamics at the free surface observed in a range of experiments [5-10]. One intriguing finding about the enhanced surface dynamics is that it is Arrhenius, in sharp contrast with the cooperative glassy dynamics usually found in the inner regions $[5,7,8]$.

Molecular dynamics simulations are instrumental in the study of glassy polymers [11]. Generic properties independent of the details in the molecular structure are most efficiently simulated by using classical coarse-grained models in which groups of closely bounded atoms are represented by point particles. Within this framework, thin films that are supported by a substrate [12-15], free standing [12,16,17], or sandwiched between impenetrable walls [18-22] were studied. Experimentally observed deviations of $T_{g}$ from the bulk value were generally reproduced. Measurement of mean square displacement of particles at free surfaces also shows much enhanced mobility [14,17]. Effects of confinement have also been studied by simulating driven flow of polymer films sandwiched between repulsive walls $[19,20]$.

This paper aims at gaining a better molecular-level understanding of the experimentally measured thin-film viscosity and mobility in Ref. [8]. In that work, the morphological evolution of initially flat unentangled short-chain polystyrene (PS) films supported by silica was monitored by atomic force microscopy [23,24]. Due to thermal activation and destabilizing film-substrate interactions, the films roughen

*C.H.Lam@polyu.edu.hk

†okctsui@bu.edu spontaneously with the development of surface capillary waves. Further evolution is dictated by drifts induced by surface tension and the film-substrate interactions, as well as thermal diffusion. By comparing the surface roughening rate with predictions based on a drift diffusion equation [25,26], the film mobility and polymer viscosity were obtained as functions of the film thickness and temperature.

In the following, we report large-scale nonequilibrium molecular dynamics simulations on the driven flow of supported polymer films. Results are compared to experiments in Ref. [8]. External forces are applied to the film particles to model the effects of pressure gradients induced by surface tension and film-substrate interactions in roughening films. From the steady-state velocities of the simulated particles, film mobility and viscosity are measured. More importantly, particle velocity profiles that are not experimentally accessible are also studied. The rest of the paper is organized as follows: In Sec. II, we explain the model and the simulation method. The results are described in Sec. III, while Sec. IV concludes the paper.

\section{SIMULATION METHOD}

We simulate a bead-spring model of polymer chains identical to that studied in Refs. [18,19]. Van der Waals interactions between particles are modeled by a truncated and shifted Lennard-Jones (LJ) potential given by

$$
U_{L J}(r)=4 \epsilon\left[\left(\frac{\sigma}{r}\right)^{12}-\left(\frac{\sigma}{r}\right)^{6}\right]-U_{0}
$$

for $r \leqslant r_{c}$ and zero otherwise. Here, $\sigma$ denotes the particle diameter, and the cutoff $r_{c}$ is given by $2 \times 2^{1 / 6}$ so that the potential has both attractive and repulsive parts. The constant $U_{0}$ follows from the continuity of $U_{L J}$ at $r_{c}$. Bonded particles are further bounded by a finitely extensible nonlinear elastic (FENE) potential [27]

$$
U_{F E N E}(r)=-\frac{k}{2} R_{0}^{2} \ln \left[1-\left(\frac{r}{R_{0}}\right)^{2}\right],
$$


where $k=30 \epsilon / \sigma^{2}$ and $R_{0}=1.5 \sigma$. It forbids the unphysical crossing of chains through each other. We adopt a dimensionless system of units where $\sigma=\epsilon=1$ and particle mass $M=1$.

The substrate is modeled by an fcc (100) surface [21,22] of lattice constant 2. The larger lattice constant allows film particles to squeeze in between the top layer of substrate atoms and provides appreciable interface roughness. This enhances the interface friction and reduces density oscillations close to the substrate. Each substrate atom has mass $M_{s}=1$ and is anchored to its lattice position by a linear spring of spring constant $k_{s}=100$. They interact with the particles via an LJ potential with $\sigma_{s}=1.2$ and $\epsilon_{s}=1.5$, while the cutoff distance remains unchanged. With these parameters, the film readily wets the substrate and the no-slip boundary condition under driven flow holds in the temperature range studied. In addition, particles are not able to penetrate into the substrate.

Our simulations are performed using the HOOMD software package [28], which runs on graphic processing units (GPUs) and provides an order of magnitude speedup over CPU-based simulations. The simulation cell has a size of $L \times L \times \infty$ under periodic boundary conditions in the lateral directions. More precisely, the cell thickness is large but finite, which makes no difference as the chains never vaporize. Two monolayers of substrate atoms are simulated with the upper layer positioned at $z=0$.

The preparation of thermalized polymer films in general follows previous techniques [18,29] with changes mainly due to programming convenience. Initial polymer chain configurations are generated by three-dimensional off-lattice random walks starting from random positions in a rectangular box of size $L \times L \times h_{I}$ placed right above the substrate, where $h_{I}$ is determined from an initial particle density of 0.85 . We first perform a push-off of overlapping particles. To avoid numerical divergence, the hard-core repulsive part of the LJ potential is temporarily replaced by a soft linear potential and the FENE potential by a harmonic potential [17]. A downward force is also applied to the particles to suppress vaporization. Molecular dynamics simulations are performed with a time step of 0.001 and temperature targeted at 0.01 using dissipative particle dynamics (DPD) applied to all particles. As push-off proceeds, the magnitude of the linear repulsive potential is increased gradually while the downward force is reduced. Towards the end of the process, we revert back to the LJ and FENE potentials and remove the downward force.

Subsequent simulations are conducted with a time step of 0.005 under NVT conditions with constant volume and temperature. Note that the simulation cell of fixed volume is tall compared with the film, the thickness of which varies with time nontrivially. A Nosé-Hoover thermostat is applied only to the substrate atoms, which has zero net motion even during forced flow. A DPD thermostat could be applied to the film particles but is switched off for better computational efficiency. After the push-off as described above, the system is annealed at $T=1$ and then at $T=0.60$ each for $10^{7}$ time steps. We then repeatedly quench and anneal the system by steps of $\Delta T=0.02$ until the desired temperature is reached. Each of these quenching or annealing processes involves $10^{7}$ time steps. At $T \geqslant 0.50$, the rms displacement of the particles during each quenching and annealing process exceeds $3 R_{g}$ in all parts of the films, where $R_{g} \simeq 1.5$ in this work. Hence the films can be considered as fully relaxed. As temperature decreases, the films are in a supercooled state. At the lowest temperature of $T=0.36$ considered here, the corresponding rms displacement of the slowest layer of particles, which resides close to the substrate, is only about 0.2 . Nevertheless, we do not observe noticeable dependence of our results on the annealing duration.

To study the film transport properties in the linear regime, we apply a small external force $f_{e}$ in the $+\hat{x}$ direction to each particle in an annealed film [19]. The forces are turned on gradually over $5 \times 10^{5}$ time steps. If they are turned on abruptly, the films may be damaged and admit poor temperature control because of degraded thermal conductance caused by defects creation. To ensure linear transport, we have checked that decreasing $f_{e}$ in less extensive runs gives a similar flow velocity profile after normalization by $f_{e}$. We find that a range of more sensitive tests is also required. Specifically, the average radius of gyration $R_{g x}$ of the chains projected on the $x$ axis should be a constant with respect to time after taking statistical fluctuations into account. Otherwise, chain deformations can speed up the flow, leading to a nonlinear flow regime in the presence of shear thinning. The density profile, especially at the surface should also be unchanged, indicating the absence of stress-induced roughening. Moreover, the instantaneous film temperatures measured from snapshots of film configurations admit a small rms fluctuation of less than 0.005 , while the time-averaged film temperature is consistent with the nominal value within \pm 0.001 in all cases. This verifies that the heat generated in the films due to the forced flow is well dissipated via the substrate. Simulations are computationally most demanding for thick films at low temperatures due to the slow flow rate. The longest run reported here takes about 12 days to execute on a Nvidia GTX580 GPU.

\section{RESULTS AND DISCUSSION}

Systems of lateral width $L=36$ and chains of length $N=10$ are considered. Our main study involves five film samples consisting of 450,600, 900, 1200, and 1800 chains, corresponding to various thicknesses $h$ between 3.0 and 13.0 (see below). Figure 1 shows the film with 900 chains after

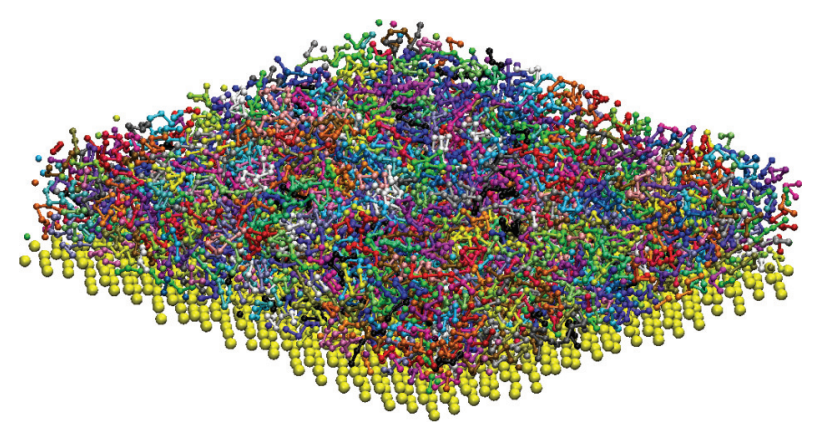

FIG. 1. (Color online) A film of 900 chains (randomly colored) of length $N=10$ on a substrate shaded in yellow (bottom) at $T=0.36$. Note that periodic boundary conditions are applied in the lateral directions. 


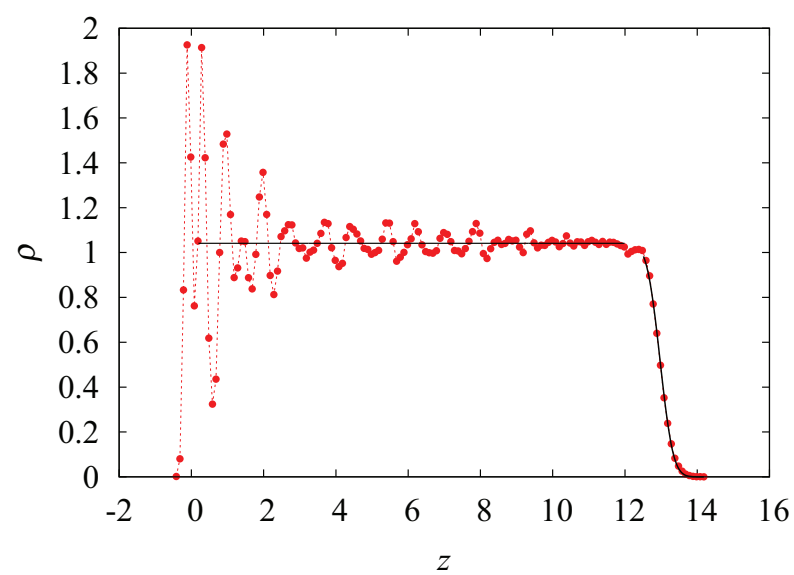

FIG. 2. (Color online) Plot of density $\rho$ against $z$ for a film with 1800 chains at $T=0.36$. The solid lines show fits to a constant and the cumulative normal distribution function for the inner and surface regions, respectively.

annealing at $T=0.36$. We calculate the density profiles of the films by histograming the $z$ coordinates of the particles. Figure 2 plots the density $\rho$ of the film with 1800 chains at $T=0.36$ averaged over the second half of the annealing process. Density oscillations are observed close to the substrate [18], which, despite decaying, extends all the way to the free surface at low temperature, as seen before [15]. These oscillations are reproducible and indicate preferred positions relative to the substrate. Nevertheless, visual inspection of the films indicates no crystallized region. The density in the inner region averages to $\rho_{0}=1.04$. At the surface, it fits well to $\rho=\rho_{0} \Phi[(h-z) / W]$ with a film height of $h=13.0$ and an rms surface width of $W=0.286$, where $\Phi$ denotes the standard cumulative normal distribution function. This form follows from the equilibrium Gaussian surface height profile [30], despite being supercooled. By conducting similar simulations and analyses, we find that the five films studied have thickness $h=3.0,4.1,6.3,8.5$, and 13.0 at $T=0.36$. For convenience, these values are used to label the nominal thickness of the films.

In this study, we are mainly interested in driven flow. Under a planar pressure gradient $\nabla P$ in the $+\hat{x}$ direction, the particles move with an average velocity $\bar{v}_{x}$ in the steady state. Consider a cross-sectional region of dimension $l_{y} \times h$ normal to the flow direction and extending over the whole thickness of the film. The volumetric flow rate equals $Q=\bar{v}_{x} l_{y} h$. We define a fluid current density per unit width of the cross-sectional region, $J=Q / l_{y}=\bar{v}_{x} h$. Considering linear transport,

$$
J=M|\nabla P|,
$$

where $M$ is the film mobility characterizing the transport efficiency of the film as a whole. Observing that a pressure gradient induces a force $f_{e}=|\nabla P| / \rho_{0}$ on each particle, one may write that

$$
M=\frac{\bar{v}_{x} h}{f_{e} \rho_{0}},
$$

where $\rho_{0}$ can be most conveniently obtained from $\rho_{2 d}=\rho_{0} h$, with $\rho_{2 d}$ denoting the areal density of the film.
We now focus on temperatures at $T=0.50,0.40$, and 0.36 , which are respectively well above, close to, and below the bulk $T_{g} \simeq 0.40$ in this model. To estimate the bulk $T_{g}$, we employ standard density measurement during cooling of bulk samples under periodic boundary and NPT conditions. The value is consistent with those found for the same model with slightly smaller values of the cutoff $r_{c}[14,16]$.

External forces equal to $f_{e}=0.002,0.005$, and 0.01 , respectively, are applied to particles in films quenched to $T=0.50,0.40$, and 0.36. Doubling $f_{e}$, for example, will lead to a measurable rise up of the film temperature for $T=0.50$ and an increase in the projected radius of gyration $R_{g x}$ for $T \leqslant$ 0.40. Molecular dynamics simulations as explained in Sec. II are conducted. Particle positions are recorded after simulating every $3000,3 \times 10^{4}$ and $10^{5}$ time steps, respectively. In each case, 10000 snapshots are recorded. All analyses are based on the last 9000 snapshots, corresponding to configurations long after the steady state is attained. At $T=0.36$, the transport is the slowest, but the total displacement of the surface particles still averages to about 20 in the $+\hat{x}$ direction, which is well beyond $R_{g}$.

We calculate the velocity $\bar{v}_{x}$ averaged over all the particles in the film and times using the particle positions snapshots. The film mobility then follows from Eq. (4), and the results are plotted in Fig. 3. Transport properties are conventionally characterized by the fluid viscosity. For a fluid with a homogeneous viscosity $\eta$ under Poiseuille flow, i.e., a simple laminar open-channel flow, with no slip at the solid boundary, we have

$$
M=\frac{h^{3}}{3 \eta},
$$

where $\eta$ is independent of $h$. However, as we will see below, the viscosity in our polymer films at low temperatures cannot be assumed homogeneous and Eq. (5) needs to be reinterpreted. Given $M$, Eq. (5) then becomes a definition of a thin-film viscosity $\eta$, which is a weighted average of the local viscosities. The hence calculated $\eta$ is plotted against the film thickness $h$ in Fig. 4.

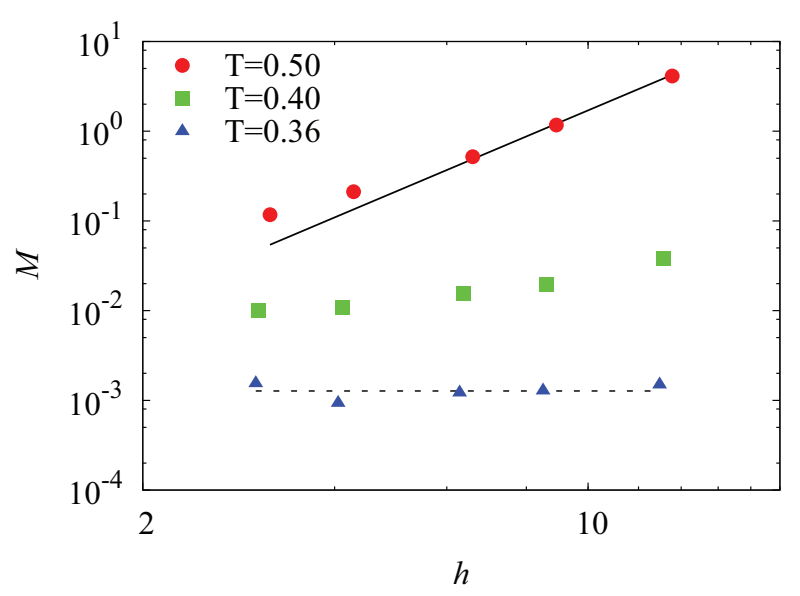

FIG. 3. (Color online) Plot of film mobility $M$ against film thickness $h$. The solid and dashed lines show fits to $M \propto h^{3}$ and a constant, respectively. 


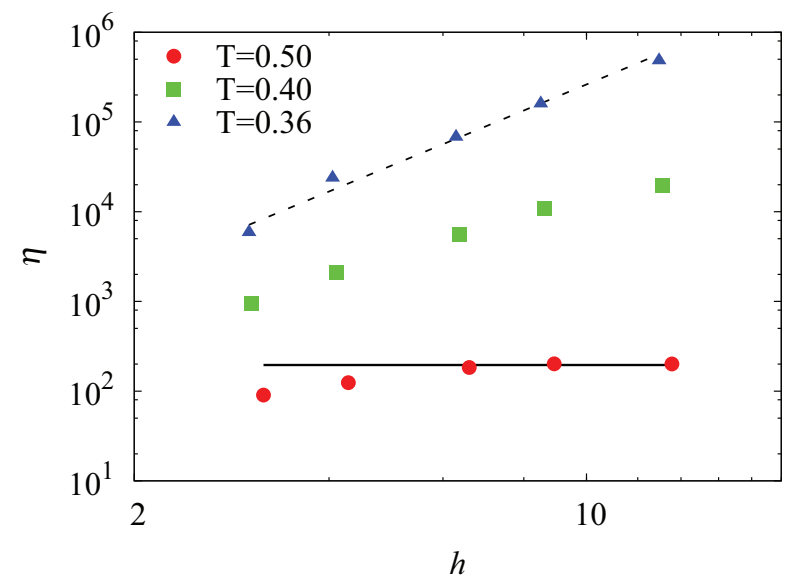

FIG. 4. (Color online) Plot of thin-film viscosity $\eta$ against thickness $h$. The solid and the dashed lines show fits to a constant and $\eta \propto h^{3}$, respectively.

From Figs. 3 and 4, one can see that the film mobility $M$ increases notably with $T$. On the other hand, $\eta$, being related to $M$ by Eq. (5), decreases with $T$ as expected. We now focus on the thickness dependence of our result. At $T=0.50$, well above the bulk $T_{g}$, and $h \geqslant 6.3$, we obtain a constant value for $\eta$, while $M \propto h^{3}$ as predicted by Eq. (5) for Poiseuille flow. Despite some deviations for $h<6.3$, the dynamics is generally well characterized by a single homogeneous viscosity $\eta$, signifying its being a material parameter.

At $T=0.36$, below $T_{g}, M$ becomes independent of $h$, indicating that Poiseuille flow with a homogeneous viscosity breaks down completely. At the same time, the thin-film viscosity $\eta$ follows $\eta \propto h^{3}$. That $\eta$ depends on $h$ implies that it is no longer an intrinsic material parameter and the local viscosity is very inhomogeneous spatially. The dynamics is then most naturally characterized by the mobility $M$ of the film. At $T=0.40$, the data show intermediate behaviors.

Experiments on PS supported by silica have demonstrated a similar constancy of $\eta$ and $M$ with respect to $h$ at high and low temperatures, respectively, via appropriate data collapses [8]. At low temperatures, the film mobility $M$ being independent of $h$ means that thinner films transport the fluid as efficiently as thicker films. This is interpreted as a signature of surface transport in Ref. [8]. We provide below direct numerical support for this interpretation.

For each snapshot of particle positions, we rank the particles according to their $z$ coordinates. The flow velocity profile is studied by computing a layer-resolved particle velocity $v_{x}$. The first layer is composed of the lowest 450 particles. The next lowest 450 particles constitute the second layer, and so on. The $i$ th data point corresponds to values of $v_{x}$ and $z$ obtained by averaging over all members in the $i$ th layer and over time, noting that the particles contained in a layer may change with time. This layering approach, in contrast with some established methods [17,18], provides the convenience of comparable statistics for each data point. Here, particle velocity $v_{x}$ is inferred from the particle displacement up to the next snapshot. Particles that have moved to other layers in the second snapshot are not excluded. We have chosen durations between snapshots such that similarly defined layer-
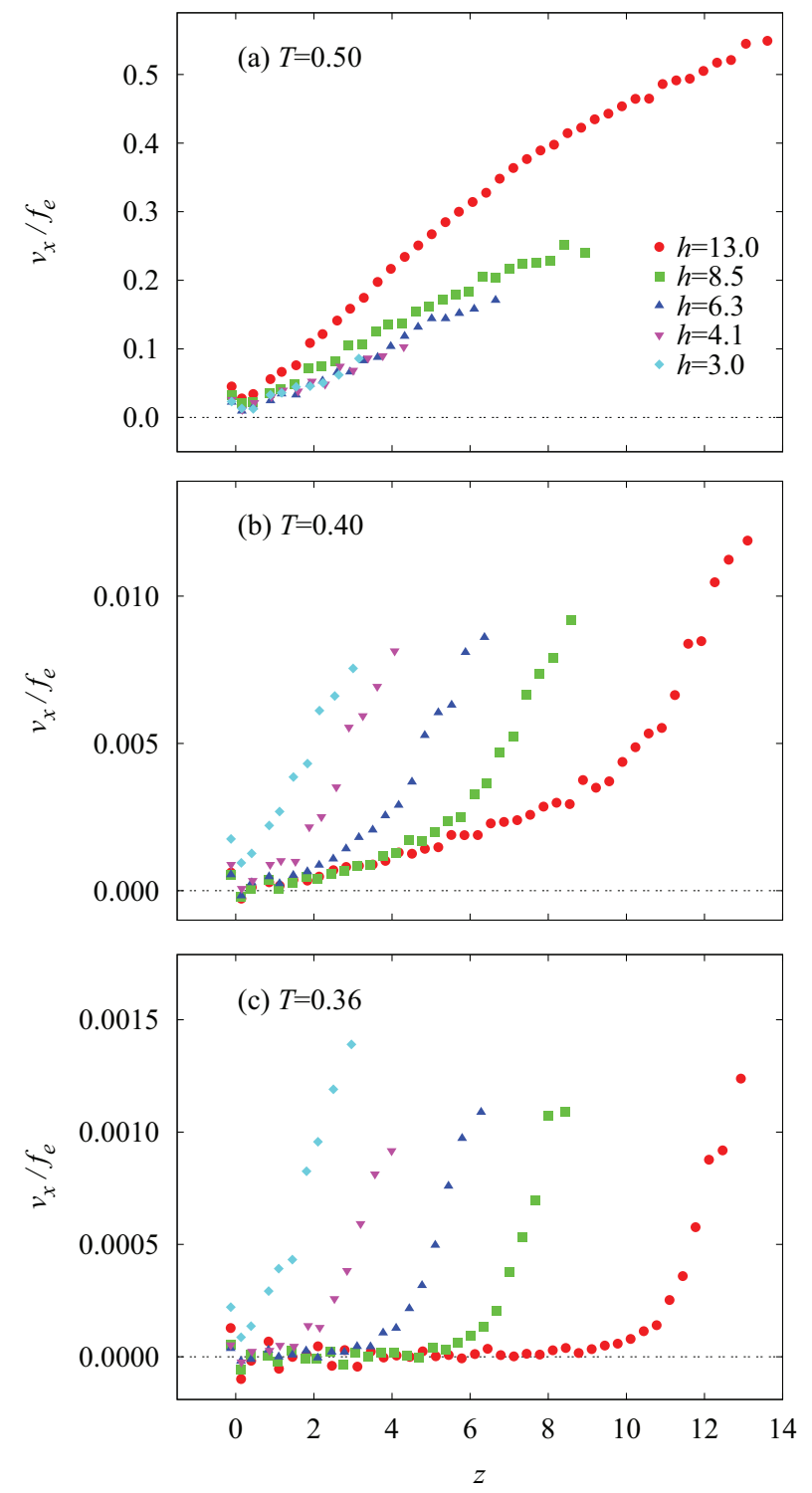

FIG. 5. (Color online) Plots of force-normalized particle velocity $v_{x} / f_{e}$ against distance $z$ from the substrate at $T=0.50$ (a), 0.40 (b), and $0.36(\mathrm{c})$.

resolved mean square displacement in the $z$ direction is less than 0.3 , even at the film surface. Intermixing between the layers over consecutive snapshots is hence small.

Figure 5 shows the force-normalized velocity profile $v_{x} / f_{e}$ as a function of $z$ found in our simulations. At $T=0.50$ [Fig. 5(a)], $v_{x}$ increases monotonically with $z$ and in general also with $h$ as expected. Poiseuille flow with a homogeneous viscosity and no-slip boundary conditions predict that

$$
v_{x}=\frac{|\nabla P|}{2 \eta}\left(2 h z-z^{2}\right)
$$

which implies

$$
\frac{v_{x}}{h^{2}}=\frac{f_{e} \rho_{0}}{2 \eta}\left[2\left(\frac{z}{h}\right)-\left(\frac{z}{h}\right)^{2}\right] .
$$

The validity of Poiseuille flow for the current model at high temperatures is well established $[19,20]$. These equations 


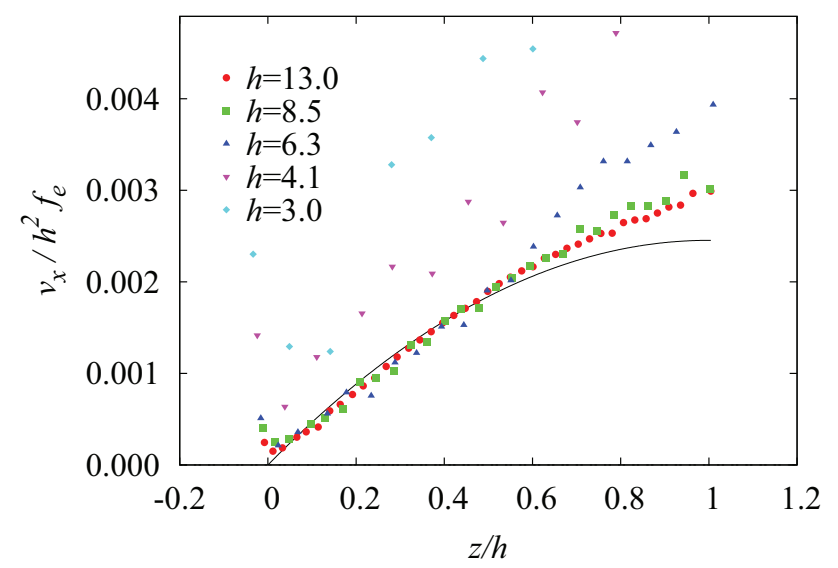

FIG. 6. (Color online) Plot of $v_{x} / h^{2} f_{e}$ against $z / h$ for $T=0.50$. The solid line shows a fit based on Poiseuille flow with a homogeneous viscosity [Eq. (7)].

describe our simulations at $T=0.60$ for sufficiently thick films as expected (data not shown). Here we illustrate the gradual breakdown of the Poiseuille description as $T$ decreases. In Fig. 6 is plotted $v_{x} / h^{2} f_{e}$ against $z / h$ using the data in Fig. 5(a) corresponding to a temperature of $T=0.50$. For $h \geqslant 6$, the three curves collapse into the same parabolic form predicted by Eq. (7) for $z / h \leqslant 0.6$. This shows the validity of Poiseuille flow and a homogeneous viscosity in the inner regions of the thicker films. For larger $z / h$, however, the velocity $v_{x}$ is faster than predicted, indicating enhanced mobility or lower local viscosity at the free surface. For the two thinner films, namely, $h=3.0$ and 4.1, the deviation from the Poiseuille form is more pronounced as the enhanced surface mobility becomes more important. The data points in Fig. 6 which deviate from Eq. (7) collapse well into a single curve when we plot $v_{x}(h)-v_{x}(z)$ against $h-z$; this implies a depth-dependent viscosity near the surface region. From Fig. 5(b), $v_{x}$ versus $z$ becomes concave at $T=0.40$, clearly distinct from the convex Poiseuille parabolic form seen in Fig. 5(a). Flow at the free surface is much enhanced, while the inner region is still able to carry considerable flow.

More interestingly, we find in Fig. 5(c) that at $T=0.36$, surface transport becomes clearly dominant as $v_{x}$ in the inner region becomes negligibly small. Figure 7 plots $v_{x}$ against the distance $h-z$ from the free surface. As seen, all the data collapse into the same curve, meaning that $v_{x}$ depends only on its distance from the free surface and is independent of the distance from the substrate. The inner region and the substrate hence play no direct role in the transport. We find that the surface flow velocity fits well to an exponential function of the form

$$
v_{x}=C f_{e} \exp \left(-\frac{h-z}{\lambda_{M}}\right),
$$

where $\lambda_{M}$ signifies a mobile layer thickness and is found to be 0.94 . We also get $C \simeq 1.63 \times 10^{-3}$.

Because of the exponential velocity profile $v_{x}$, the surface region crosses over into the inner, slower region gradually rather than abruptly, which has often been assumed in previous two-layer model analysis for simplicity [8,31]. Since an exponential profile is another simple possibility, it was

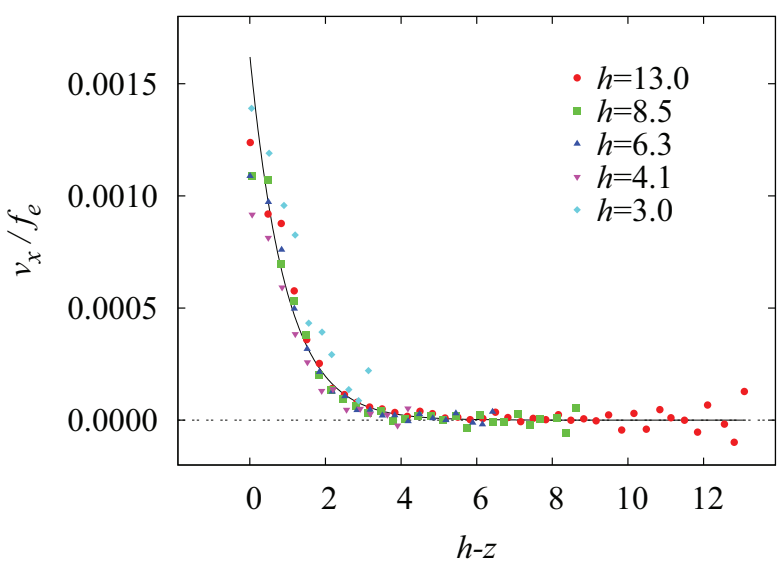

FIG. 7. (Color online) Plot of $v_{x} / f_{e}$ against distance $h-z$ from the free surface. The solid line shows a fit to an exponential function.

compared with a two-layer model recently [32]. Here we use the "skin depth" $\lambda_{M}=0.94$ to quantitatively characterize the mobile layer thickness for the observed fluid flow, although the enhanced mobility extends much deeper than $\lambda_{M}$ in the presence of the exponential tail. This value is close to the particle diameter but is also in the same order of magnitude as $R_{g} \simeq 1.5$ due to the small chain length of $N=10$ considered here. We have also performed simulations at $N=20$, which is computationally much more challenging due to the reduced flow rate and the worsened problem of shear thinning. Our preliminary result shows a much reduced mobility but a similar exponential profile with the same value of $\lambda_{M}$. We hence believe that $\lambda_{M}$ is dictated by the particle diameter rather than $R_{g}$. Furthermore, although the mobile layer thickness for flow dynamics has not been directly measured in experiments, it has been inferred to be $3 \mathrm{~nm}$ or thinner, as no significant reduction of surface transport is observed at this thickness [8].

A theoretical description of the surface mobile layer is at present controversial [33-35]. The identification of an exponential velocity profile here may contribute to scrutinizing theoretical approaches. Despite lacking a quantitative theory, much about the chain dynamics in the mobile layer can be understood qualitatively by viewing movies depicting the dynamics of individual chains [36]. It is readily observable that in the surface flow regime, there exists no sharp boundary between the actively hopping surface particles and the basically vibrating inner ones. The drift motions of the particles induced by external forces are very intermittent and are superimposed with vigorous diffusional motions. Specifically, a chain moves suddenly and quickly in the flow direction when the whole chain diffuses to regions within a multiple of $\lambda_{M}$ from the free surface. When some members of the chain randomly dive deeper into the film, the rest of the chain then appears to diffuse only back and forth around the more frozen members.

Driven flow is simulated in this work in the small driving force limit corresponding to the linear response regime. We have checked that standard quantitative measures such as the density profile, radius of gyration, layer-resolved mean square displacement in directions perpendicular to the flow, and so on are unaffected. Furthermore, during cooling of the films, the inner regions gradually become frozen into the glassy state. The dynamics then slows down dramatically, 
but many static properties are simply frozen snapshots of their higher temperature values. Chain configurations and many other related properties of the films studied here are hence qualitatively similar to those extensively reported in the literature [12-22].

\section{CONCLUSION}

In summary, we have simulated the driven flow of supported polymer films. Well above the bulk $T_{g}$, the dynamics is consistent with Poiseuille flow with a homogeneous fluid viscosity. At lower temperatures, the flow is increasingly dominated by a mobile region near the free surface. At temperatures sufficiently below $T_{g}$, the transport is completely dominated by the surface mobile region, as the contribution of the inner region becomes negligibly small. The local viscosity is therefore highly inhomogeneous. We further observe that the surface mobile region has a velocity profile that decays exponentially into the inner region of the film, with a decay width similar to the individual particle diameter.

\section{ACKNOWLEDGMENTS}

C.H.L. is thankful for support from HK PolyU via Grant No. G-YJ71. O.K.C.T. is grateful for the support of the National Science Foundation through Grants No. DMR-1004648 and No. DMR-1310536.
[1] M. D. Ediger and P. Harrowell, J. Chem. Phys. 137, 080901 (2012).

[2] J. L. Keddie, R. A. L. Jones, and R. A. Cory, Europhys. Lett. 27, 59 (1994).

[3] J. A. Forrest, K. Dalnoki-Veress, J. R. Stevens, and J. R. Dutcher, Phys. Rev. Lett. 77, 2002 (1996).

[4] S. Kawana and R. A. L. Jones, Phys. Rev. E 63, 021501 (2001).

[5] T. Kajiyama, K. Tanaka, N. Satomi, and A. Takahara, Macromolecules 31, 5150 (1998).

[6] C. J. Ellison and J. M. Torkelson, Nat. Mater. 2, 695 (2003).

[7] Z. Fakhraai and J. A. Forrest, Science 319, 600 (2008).

[8] Z. Yang, Y. Fujii, F. K. Lee, C. H. Lam, and O. K. C. Tsui, Science 328, 1676 (2010).

[9] D. Qi, M. Ilton, and J. A. Forrest, Eur. Phys. J. E 34, 56 (2011).

[10] K. Paeng, S. F. Swallen, and M. D. Ediger, J. Am. Chem. Soc. 133, 8444 (2011).

[11] J. L. Barrat, J. Baschnagel, and A. Lyulin, Soft Matter 6, 3430 (2010).

[12] J. A. Torres, P. F. Nealey, and J. J. de Pablo, Phys. Rev. Lett. 85, 3221 (2000).

[13] A. R. C. Baljon, M. H. M. Van Weert, R. B. DeGraaff, and R. Khare, Macromolecules 38, 2391 (2005).

[14] S. Peter, H. Meyer, and J. Baschnagel, J. Polym. Phys. B 44, 2951 (2006).

[15] P. Z. Hanakata, J. F. Douglas, and F. W. Starr, J. Chem. Phys. 137, 244901 (2012).

[16] H. Morita, K. Tanaka, T. Kajiyama, T. Nishi, and M. Doi, Macromolecules 39, 6233 (2006).

[17] S. Peter, H. Meyer, J. Baschnagel, and R. Seemann, J. Phys.: Condens. Matter 19, 205119 (2007).
[18] F. Varnik, J. Baschnagel, and K. Binder, Phys. Rev. E 65, 021507 (2002).

[19] F. Varnik and K. Binder, J. Chem. Phys. 117, 6336 (2002).

[20] J. Zhang, J. S. Hansen, and B. D. Todd, J. Chem. Phys. 126, 144907 (2007).

[21] J. Servantie and M. Müller, Phys. Rev. Lett. 101, 026101 (2008).

[22] N. V. Priezjev, Phys. Rev. E 82, 051603 (2010).

[23] Z. Yang, C. H. Lam, E. DiMasi, N. Bouet, J. Jordan-Sweet, and O. K. C. Tsui, Appl. Phys. Lett. 94, 251906 (2009).

[24] Z. Yang, D. Peng, A. Clough, C. H. Lam, and O. K. C. Tsui, Eur. Phys. J.: Spec. Top. 189, 155 (2010).

[25] C. H. Lam, O. K. C. Tsui, and D. Peng, Langmuir 28, 10217 (2012).

[26] H. Y. Deng, R. N. Li, H. Hunag, O. K. C. Tsui, and C. H. Lam, Langmuir 29, 4283 (2013).

[27] K. Kremer and G. S. Grest, J. Chem. Phys. 92, 5057 (1990).

[28] J. A. Anderson, C. D. Lorenz, and A. Travesset, J. Comp. Phys. 227, 5342 (2008).

[29] R. Auhl, R. Everaers, G. S. Grest, K. Kremer, and S. J. Plimpton, J. Chem. Phys. 119, 12718 (2003).

[30] See, e.g., Statistical Mechanics of Membranes and Surfaces, 2nd ed., edited by D. Nelson, T. Piran, and S. Weinberg (World Scientific, Singapore, 2003).

[31] Z. Yang, A. Clough, C. H. Lam, and O. K. C. Tsui, Macromolecules 44, 8294 (2011).

[32] J. E. Pye, K. A. Rohald, E. A. Baker, and C. B. Roth, Macromolecules 43, 8296 (2010).

[33] S. Herminghaus, Eur. Phys. J. E 8, 237 (2002).

[34] D. Long and F. Lequeux, Eur. Phys. J. E 4, 371 (2001).

[35] S. T. Milner and J. E. G. Lipson, Macromolecules 43, 9865 (2010).

[36] Chain dynamics movies available at http://apricot.ap.polyu.edu.hk/flow. 